\title{
International agbio consortium takes shape in Beijing
}

\author{
Rafael Rangel-Aldao
}

Mobilizing biotechnology to address crises in human nutrition and agricultural productivity remains problematic. Most current agricultural commercial biotechnology efforts are overwhelmingly directed at developed-world problems, with little impetus or incentive to focus on the needs of poorer nations.

With this in mind, a recent meeting in Beijing* considered the feasibility of forming a consortium of scientists to develop and share biotechnologies to improve the yield and quality of key crops for developing countries.

Speakers from every corner of the globe were represented-from institutes and laboratories in North and South America, South Africa, Europe, and Asia-all united by a solid record of working in international partnerships. Alejandro Mentaberry from the University of Buenos Aires, Argentina, presented the results of a group of 13 Latin American and European countries, established by the Ibero-American Program of Science and Technology for Development (CYTED, Madrid, Spain), for the transformation of potato indigenous cultivars with a set of 16 genetic constructs carrying different two-gene combinations.

Transformation vectors were designed in such a way that a maximum of six genes could be introduced in the same plant using different markers. Transformants expressing combinations of two and four genes such as lysozyme and attacine (from the giant silk moth) resulted in bacterial resistance in field tests to Erwinia, an important pest in Chile and Brazil. Similarly, different combinations of antiviral genes (i.e., for PVY coat protein and PLRV coat protein or replicase) and antifungal genes (i.e., for ribosome inhibitor protein, chitinase, AP24 and glucanase) are currently being tested in field trials in Chile, Argentina, Uruguay, Cuba, Brazil, and Spain.

Dave Berger from ARC-Roodeplaat Vegetable and Ornamental Plant Institute in Pretoria, South Africa, presented data from multidisciplinary teams that have accumulated experience throughout the

Rafael Rangel-Aldao is director of $R \& D$, Centro Tecnológico Polar, Empresas

Polar, Caracas, Venezuela

(rrangel@empresas-polar.com). agbiotechnology cycle-from gene isolation to transgenic field tests-in order to target local agricultural diseases that are not necessarily important outside that region. For example, South African potato cultivars were transformed for resistance to potato leafroll virus and tested in the first field trial of transgenic potato in Africa.

Atanas Atanassov, from the Institute of Genetic Engineering in Kostinbrod, Bulgaria, described his institute's efforts to strengthen crop breeding in Bulgaria as the first country to commercialize plant biotech varieties in Europe, and gave examples of transformed wheat, barley, rice, sugar beet, sunflower, alfalfa, and tobacco with improved agronomic traits.

Zhanglian Chen, from the College of Life Sciences at Peking University in Beijing, China, highlighted the major areas of interest to China in transgenic plants: resistance to disease, insects, herbicides, and stress; as well as yield and quality improvement of seeds. Chen gave specific examples: field trials of insect-resistant cotton (mostly from Monsanto) in the Xinjiang province; field trials for virusresistant pepper transformed with the gene for CMV coat protein; virus-resistant rice transformed with the coat protein gene of RDV; and color-altered petunias transformed with the ChsA gene. Many of these applications have already received approval for commercialization in China.

These and other examples of international, regional, and national collaborations in plant biotechnology encouraged us to create an alignment of our various research centers, programs, and universities into a global consortium organized within the International Center for Genetic Engineering and Biotechnology (ICGEB, Trieste, Italy) as a vehicle for cooperation among more than 40 countries.

The specific agenda of the consortium is as follows: (1) To develop new biotechnological applications for crops resistant to disease, herbicides, insects, and abiotic stress, as well as those directed to increase food quality and yield, with particular emphasis devoted to gene discovery through the exploration and exploitation of local biodiversity. The principal target crops will be rice, potato, maize, cotton, and cassava. (2) To promote research in basic science, in particular, in stress signal transduction and genomics. (3) To encourage new systems of biotechnological applications (e.g., inte- grated pest management). (4) To address by means of responsible public forums the societal implications of agricultural biotechnology (socioeconomics, biosafety, intellectual property rights, preservation of biodiversity).

The plan for realizing this agenda is multistaged. Level 1 is a forum for the exchange of scientific, technological, and system information in agricultural biotechnology as it applies to the development of appropriate applications in the various participating countries. Members of the forum will meet annually at the ICGEB. Level 2 comprises material exchange between members of genes, vectors, gene constructions, promoters, etc., as is currently done within the CYTED network. Level 3 would promote the exchange of scientists, students, and technicians between member countries to further their working knowledge of the techniques of modern plant biotechnology. Level 4 will implement the formation of multinational groups working on a particular research project or biotechnological application, and level 5 will bring collaboration in field trials, environmental impact studies, as well as evaluation of yields, qualities, etc. Finally, level 6 will include the introduction of transgenic crops with improved traits.

The consortium, once formalized, will comprise a new, and hopefully newly empowered, grouping of scientists concerned with developing and implementing the technologies necessary to increase world food production. It will also provide an infrastructure to allow cascade-type amplification for experimentation with new genes, from basic research laboratories to national plant breeding institutes, international centers of germplasm and plant breeding, and national programs for the application of agricultural biotechnology.

*"Research and Cooperation Activities in Plant Biotechnology," held May 19-21 in Beijing. The meeting was co-organized by the author and Xiaocheng Gu of the National Laboratory of Protein Engineering and Plant Genetic Engineering at Peking University, Beijing. It was sponsored by the International Center of Genetic Engineering and Biotechnology (ICGEB, Trieste, Italy; and New Delhi, India), the Ibero-American Program of Science and Technology for Development (CYTED, Madrid, Spain), the International Center of Tropical Agriculture (CIAT, Cali, Colombia), and several governmental agencies from China, including the Ministry of Science and Technology and the China National Centre for Biotechnology and Development. 